\title{
Estudo e implementação de um arranjo de microfones para a estimação da direção de chegada
}

\author{
Julia S. de Moraes e Silva , José A. Apolinário Jr. e Marcello L. R. de Campos
}

\begin{abstract}
Resumo-Este artigo apresenta detalhes de implementação e resultados do emprego de algoritmos de estimação de direção de chegada (DOA) num arranjo linear de microfones. Com este arranjo de 8 microfones, usando um conversor AD com pré-amplificadores embutidos, adquirimos o sinal por meio do software Matlab ${ }^{\mathbb{B}}$ onde é feito um processamento do sinal para sua adequação à condição de sinal analítico de banda estreita. Foram implementados dois métodos conhecidos de estimação de direção de chegada: o Beamforming e o Capon. O objetivo do trabalho é comparar tais métodos visando a uma aplicação em uma vídeo conferência onde uma câmera de vídeo é comandada a partir do DOA estimado.
\end{abstract}

Palavras-Chave-Arranjo de microfones, direção de chegada, processamento de sinais em arranjos de sensores, DOA.

Abstract-This paper presents details of implementation and results of employment of algorithms for estimating the direction of arrival in a linear array of microphones. With this array of 8 microphones and an AD converter with built-in preamplifiers, we acquired and processed the signal using the software Matlab ${ }^{\mathbb{R}}$ in order to rendering it a narrow band analytical feature. We implemented two known methods of estimating the direction of arrival: Beamforming and Capon. The objective of the study is to compare such methods in a Video conference application, where a video camera will be controlled by the estimated DOA.

Keywords-Microphone array, direction of arrival, sensor array signal processing, DOA.

\section{INTRODUÇÃO}

Este trabalho aborda um tema típico da área de antenas inteligentes, a estimação da direção de chegada (direction of arrival, DOA, como é conhecido) [1]. Os mesmos algoritmos são, contudo, usados em arranjos de qualquer tipo de sensores (antenas, microfones e hidrofones são alguns exemplos). Visando a sua aplicação em vídeo conferência, implementamos um arranjo linear uniforme (ULA, do inglês uniform linear array) com oito microfones. Este trabalho, desenvolvido conjuntamente nos laboratórios de processamento de sinais do IME e da UFRJ, busca ainda apresentar uma plataforma de estudos de processamento de sinais em arranjos de sensores utilizando equipamentos e componentes de fácil aquisição no mercado nacional e de ampla utilização acadêmica. Nas próximas seções, detalharemos o arranjo, o experimento conduzido, seus resultados e conclusões.

\section{Fundamentos e detalhes de implementaÇão}

A implementação de um arranjo genérico deve considerar o tipo e a disposição dos microfones utilizados. Aconselha-se o uso de microfones omnidirecionais ou cardióides, pois a diretividade excessiva pode comprometer a aquisição do sinal, e a disposição linear dos microfones, pois facilita a análise espacial. Um dos fatores de maior relevância é o estágio de pré-amplificação. Necessita-se manter as características de fase do sinal, a fim de se determinar a direção de chegada. Portanto, os pré-amplificadores não devem introduzir atrasos de fase. O hardware FIREPOD é uma interface de gravação equipada

Julia Simões de Moraes e Silva e Marcello Luiz Rodrigues de Campos pertencem ao Departamento de Eletrônica da Universidade Federal do Rio de Janeiro (UFRJ) e José Antonio Apolinário Jr. ao Departamento de Engenharia Elétrica (SE/3) do Instituto Militar de Engenharia (IME), Rio de Janeiro, Brasil. E-mails: juliasimoes@ufrj.br, apolin@ime.eb.br, mcampos@ieee.org. Este trabalho foi financiado pelo CNPq (PIBIC-IME 2007/2008) e pelo EB/DCT (PBCT 2007) com 8 pré-amplificadores para microfones de $24 \mathrm{bit} / 96 \mathrm{kHz}$ com baixo nível de ruído, alto headroom nos pré-amplificadores e phantom power $(+48 \mathrm{v})$. No projeto, usamos o software Matlab ${ }^{\circledR}$ para fazer o processamento do sinal. Ele foi escolhido por motivos didáticos. Decidimos usá-lo também para fazer a aquisição dos sinais; sendo assim, usamos somente um programa para todo o projeto. A aquisição de dados é feita com o pacote pa_wavplay para o Matlab ${ }^{\circledR}$, que grava e reproduz sinais multicanais. Para fazer as gravações temos que fazer o download do pacote pa_wavplay e salvá-lo no diretório principal do Matlab ${ }^{\circledR}$. Devemos também adicionar seu path no arquivo de path do Matlab ${ }^{\circledR}$, em File-SeePath adicione o diretório onde está o pacote. Para gravar, usamos o comando pa_wavrecord que retorna uma matriz com o número de colunas de acordo com o número de canais desejados e o número de linhas com o número de amostras escolhido.

$\mathrm{O}$ arranjo linear uniforme possui esta denominação pois os sensores estão dispostos sobre uma mesma reta e o espaçamento entre eles é constante e igual a $d$. As frentes de ondas serão consideradas planas (hipótese fun-field) e $d \leq \frac{\lambda}{2}$ [4]. Este último possui um efeito similar ao que ocorre no caso de se trabalhar com freqüência de amostragem (no tempo) abaixo da necessária. O objetivo desta modelagem é relacionar os sinais na saída deste arranjo de sensores com a direção chegada do sinal. Estas saídas são as informações disponibilizadas pelo sistema; busca-se estimar a direção de chegada a partir das amostras recebidas em cada sensor [2].

\section{Sobre os ALgORITMOS UTILIZADOS}

No projeto foram usados dois métodos conhecidos de estimação de direção de chegada: Capon e Beamforming. Ambos têm como características básicas: assumem conhecido o steering vector, $\mathbf{a}(\theta)$, não fazem suposição sobre propriedades estatísticas do sinal (são considerados não-paramétricos) e se baseiam no princípio de que um filtro espacial pode ajustar seus coeficientes para ressaltar o sinal proveniente de uma determinada direção [1].

No caso do Beamforming ${ }^{1}$, podemos atrasar os sinais dos sensores de forma que sua soma seja construtiva para uma dada direção. Para isso, definimos abaixo o steering vector determinado pela geometria do arranjo de sensores. Para um ULA de 8 sensores, temos:

$$
\mathbf{a}(\theta)=\left[\begin{array}{lllll}
1 & e^{-j \frac{\omega d \sin \theta}{v}} & e^{-j \frac{\omega d \sin \theta}{v} 2} & \ldots & e^{-j \frac{\omega d \sin \theta}{v} 7}
\end{array}\right]^{T} .
$$

Onde, $\omega$ é a freqüência do sinal, $v$ é a velocidade da luz e $\theta$ o ângulo desejado.

Para um filtro espacial com vetor de coeficientes $\mathbf{h}$, a potência do sinal de saída é dada por

$$
P=E\left[\left|\mathbf{h}^{H} \mathbf{x}(t)\right|^{2}\right],
$$

onde $\mathbf{x}$ corresponde ao vetor contendo os sinais dos sensores, o qual é assumido igual a $\mathbf{a}^{T}(\theta) x(t)$, possivelmente corrompido com ruídos e interferidores (sinais de outras direções).

\footnotetext{
${ }^{1}$ Beamforming neste contexto é uma técnica de estimação de DOA; não confundir com o outro emprego clássico de arranjos de sensores que leva o mesmo nome e é conhecido em português como conformação de feixe.
} 
Assumindo que desejamos que o sinal proveniente de uma dada direção (dada por $\mathbf{a}(\theta)$ ) passe sem distorção e que os sinais de todas as demais direções sejam atenuados, podemos minimizar a potência da saída sujeito à restrição $\mathbf{h}^{H} \mathbf{a}(\theta)=1$. Tal critério, se inicialmente considerarmos sinais espacialmente brancos (proveniente igualmente de todas direções) no cômputo de $\mathbf{h}$, chegamos a uma expressão para a potência da saída proporcional à mostrada na equação seguinte e a partir da qual levantamos seus picos em função do ângulo $\theta$ como possíveis direções de chegada, sendo $\mathbf{R}$ a matriz de correlação do vetor de sinais de entrada:

$$
\mathbf{a}^{T}(\theta) \mathbf{R a}(\theta) .
$$

Por outro lado, o método de Capon objetiva estimar a potência na direção de interesse, anulando ou minimizando a interferência nas demais direções. De forma similar ao Beamforming, busca-se pelo máximo da expressão seguinte, o que corresponde a minimizar $\mathbf{h}^{H} \mathbf{R h}$ sujeito a $\mathbf{h}^{H} \mathbf{a}(\theta)=1$ :

$$
\frac{1}{\mathbf{a}^{T}(\theta) \mathbf{R}^{-1} \mathbf{a}(\theta)} .
$$

\section{DESCRIÇÃO DO EXPERIMENTO}

O arranjo é constituído de uma base de acrílico com suporte para oito microfones (foram utilizados microfones da marca Behringer, modelo XM8500 Ultravoice). A digitalização do sinal e a conexão do arranjo é feita com uma placa de aquisição de audio Firepod da Presonus. O Firepod tem conexão firewire, encontrada na maioria dos computadores atuais, o que tornou o arranjo mais portátil. A distância entre os microfones foi fixada em $10 \mathrm{~cm}$. O sinal usado para teste é um tom de $1,7 \mathrm{kHz}$ — esta frequiência foi escolhida respeitando o teorema da amostragem espacial para a distância de entre microfones de $d=10 \mathrm{~cm}$ (igual a $\lambda / 2$ para esta freqüência)—assumido de banda estreita da forma $x(t)=s(t) e^{j w t}$. Entretanto, o sinal real não apresenta esta característica. Fez-se necessário um tratamento no sinal para permitir o atraso por meio da multiplicação por uma exponencial complexa. Como o sinal real é uma cossenóide, sabemos que $\cos (w t)=\frac{e^{j w t}+e^{-j w t}}{2}$, ou seja, temos um espectro simétrico em relação à origem (para $\omega<0$ ) o qual poderá ser eliminado (tornando o espectro do sinal modulado tal como desejamos) por meio do seguinte procedimento:

1. Multiplicamos $x(t)$ por 2;

2. Construímos dois filtros: um passa-baixas com frequiência de corte $\omega=0.5 \pi$ o qual é transladado para a direita pela multiplicação pela exponencial complexa $e^{j 0.5 \pi n}$. O outro filtro passa-banda centrado na freqüência do sinal e com uma largura de banda de $200 H z$;

3. Convoluímos então os dois filtros para criar o filtro necessário para eliminarmos a porção do espectro de freqüência de sinal negativo bem como filtrarmos ruídos fora da banda de interesse.

$\mathrm{O}$ experimento pode então usar este sinal tratado para fazer a estimação da direção de chegada com os cálculos apresentados anteriormente. Desenvolvemos um programa em Matlab para efetuar todos os cálculos para o tratamento dos sinais dos microfones e a estimação da direção de chegada, além de efetuar também a aquisição do sinal. O resultado, para um sinal desejado chegando ao arranjo com 0 grau, é apresentado na Figura 1.

Nesta figura, é possível observar os dois máximos no ângulo $0^{\circ}$ como esperado. Durante a gravação existem alguns cuidados que devem ser tomados. Todos os microfones devem estar alinhados e com a mesma distância entre si. É importante que o tom a ser observado esteja sendo bem reproduzido. Devemos também não deixar o gerador do sinal e os microfones muito próximos, pois assim não teremos frentes de onda planas chegando nos microfones.

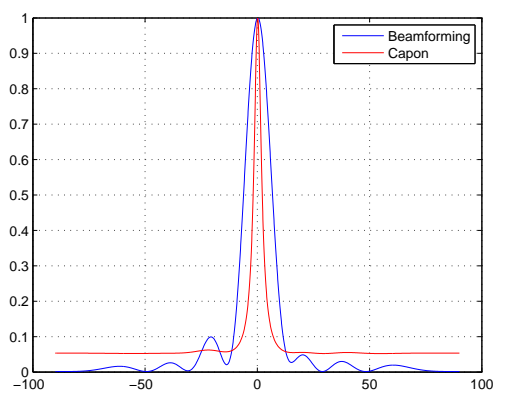

Fig. 1. Resultado do algoritmo para um ângulo de $0^{\circ}$.

A tabela I apresenta os resultados de DOA para alguns ângulos testados.

TABELA I

\begin{tabular}{|c|c|c|}
\hline Ângulo esperado & DOA & Erro \\
\hline \hline $0^{\circ}$ & 0.3 & 0.3 \\
\hline $15^{\circ}$ & 15.2 & 0.2 \\
\hline $30^{\circ}$ & 27.6 & 2.4 \\
\hline $45^{\circ}$ & 39.3 & 5.7 \\
\hline $60^{\circ}$ & 53.4 & 6.6 \\
\hline$-15^{\circ}$ & -15.9 & 0.9 \\
\hline$-30^{\circ}$ & -33 & 3 \\
\hline$-45^{\circ}$ & -44.8 & 0.2 \\
\hline$-60^{\circ}$ & -59.1 & 0.9 \\
\hline
\end{tabular}

O erro observado nas medições são devidos, principalmente, a problemas práticos: as gravações feitas em laboratório a distância entre fonte e microfones não é a ideal (em torno de $3 \mathrm{~m}$ ) para a aproximação de frentes de ondas planas, o alinhamento dos microfones foi feito visualmente e a medição do ângulo esperado foi feita também visualmente, com o auxílio de um transferidor. Contudo, os erros relativos observados foram baixos.

\section{CONClusões}

A pré-amplificação é um ítem crítico e pode, pela inserção de erros de fase, interferir no desempenho do algoritmo. Inicialmente, usamos pré-amplificadores não profissionais que causaram interferências nos resultados. Como solução usamos um amplificador profissional, fácil de ser encontrado no mercado nacional, com entrada para oito canais, o Firepod. Este amplificador também foi a solução para outra dificuldade, a locomoção do equipamento. Com a nova placa de amplificação e digitalização com conexão firewire podemos usar computadores portáteis. Os imprevistos enfrentados foram de bom proveito para o aprendizado e o conhecimento da prática da pesquisa científica, motivando a aluna para buscar conhecimento de outras áreas não contempladas no projeto original. Para a apresentação do trabalho, espera-se contar com um dispositivo que possa ser comandado diretamente pelo computador que processa os sinais, possivelmente um dispositivo contendo um motor de passo e uma conexão bluetooth.

\section{REFERÊNCIAS}

[1] Petre Stoica and Randolph Moses, Introduction to Spectral Analysis, Prentice Hall, 1997.

[2] Costa, João Paulo C. L. da, Desenvolvimento de Modelos, Técnicas e Aplicações para Arranjos de Sensores, Universidade de Brasília, Faculdade de Tecnologia, 2006.

[3] H. L. Van Tress, Optimum Array Processing, Wiley-Insterscience, 2002.

[4] Lal Chand Godara, Smart Antennas, ACRC Press, 2004. 